\title{
Theory for the Doping Dependence of Spin Fluctuation Induced Shadow States in High- $\mathbf{T}_{c}$ Superconductors
}

\author{
M. Langer, J. Schmalian, S. Grabowski, and K.H. Bennemann \\ Institut für Theoretische Physik, Freie Universität Berlin, Arnimallee 14, \\ 14195 Berlin, Germany
}

(October 4, 1995)

\begin{abstract}
We analyze the doping dependence of the intensity and energetical position of shadow states in high $-\mathrm{T}_{c}$ superconductors within the $2 \mathrm{D}$ Hubbard model and using our recently developed numerical method for the self consistent summation of bubble and ladder diagrams. It is shown that shadow states resulting from short range antiferromagnetic correlations occur for small but finite excitation energies which decrease for decreasing doping, reflecting a dynamically broken symmetry with increasing lifetime. Simultanously, the intensity of these new states increases, the quasiparticle dispersion is strongly flattened, and a pseudogap in the density of states occurs. Finally, we discuss the importance of flat bands at the Fermi level and nesting of the Fermi surface as general prerequisites for the observability of shadow states.
\end{abstract}


The electronic excitation spectrum of high- $\mathrm{T}_{c}$ superconductors and its relation to short range antiferromagnetic correlations are currently of special interest, since it reveals important information about the possibility of a spin fluctuation induced pairing mechanism. Here, the observation of shadows of the Fermi surface (FS) for $\mathrm{Bi}_{2} \mathrm{Sr}_{2} \mathrm{CaCu}_{2} \mathrm{O}_{8+\delta}$ ? by Aebi et al. is intensively debated目. Using a new experimental technique to perform a FS mapping, Aebi et al. observed besides the main FS a shadow with lower intensity which is shifted by the wave vector $\mathbf{Q}=(\pi, \pi)$ with respect to the main FS. Due to the pro-

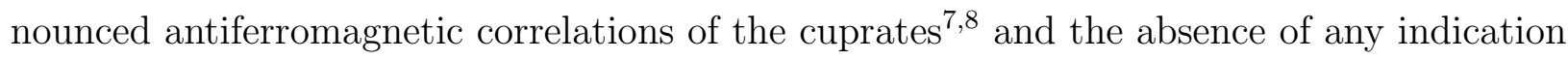
of a structural origin of these new states in LEED experiments $\$$, the corresponding $(2 \times 2)$ superstructure is believed to be of antiferromagnetic origin 1 . 6 . Nevertheless, it is a priori not clear whether the antiferromagnetic correlations with rather short correlation length are sufficient to produce new states, which one would have expected only for systems with long range ordert. Therefore, important information about the magnetic origin of the shadow states might result from an experimental as well as theoretical investigation of the doping dependence of the shadow band intensity.

In this paper we present our results for the doping dependence of the spectral density and consequently of the shadow states using a new numerical method for the self consistent solution of the fluctuation exchange approximation (FLEX) 10 of the one-band Hubbard model on the real frequency axis. We show that the intensity and the lowest energy distance of the shadow states with respect to the Fermi level are closely intertwined and depend sensitively on the doping concentration. This is interpreted as a dynamical symmetry breaking where the lifetime of the symmetry broken state increases for decreasing doping concentration. Consequently, we argue that in the cuprates the transition from the paramagnetic to the antiferromagnetic state occurs on finite time scales rather gradually, although a sharp phase transition is still observable for even lower doping concentrations.

In order to achieve an appropriate description of the low energy spin fluctuations we use in the following the fluctuation exchange approximation (FLEX) introduced by Bickers and Scalapinden. This self consistent summation of all bubble and ladder diagrams is a 
conserving approximation in the sense of Baym and Kadanofft1. In particular, it takes into account the interaction of the quasi particles with spin fluctuations which are expected to be the dominating low energy excitations in the high- $T_{c}$ materials.

We consider the one-band Hubbard Hamiltonian

$$
H=\sum_{i j \sigma}\left(t_{i j}-\mu \delta_{i j}\right) c_{i \sigma}^{\dagger} c_{j \sigma}+U \sum_{i} c_{i \uparrow}^{\dagger} c_{i \uparrow} c_{i \downarrow}^{\dagger} c_{i \downarrow}
$$

where, $c_{i \sigma}^{\dagger}$ is the creation operator of an electron at lattice site $i$ and with spin $\sigma . t_{i j}$ is the hopping matrix element between sites $i$ and $j, \mu$ is the chemical potential and $U$ is the local Coulomb repulsion. The results presented below are obtained for nearest neighbor hopping $t=0.25 \mathrm{eV}, U=4 t$ and temperature $T=63 \mathrm{~K}$. The self-energy of this Hamiltonian within the FLEX-approximation, neglecting the particle-particle excitations which were shown to be of minor importance 1 , is given by the following momentum and Matsubara-frequency $\operatorname{sum}^{\mathrm{\theta}}$

$$
\Sigma_{\mathbf{k}}\left(i \omega_{n}\right)=\frac{T}{N} \sum_{\mathbf{k}^{\prime}, n^{\prime}} G_{\mathbf{k}^{\prime}}\left(i \omega_{n^{\prime}}\right) V_{\mathbf{k}-\mathbf{k}^{\prime}}\left(i \omega_{n}-i \omega_{n^{\prime}}\right),
$$

where, $N$ is the number of lattice sites. The effective interaction $V_{\mathbf{k}}\left(i \nu_{m}\right)$ resulting from the summation of bubble and electron-hole ladder diagrams is given by

$$
V_{\mathbf{q}}\left(i \nu_{m}\right)=\frac{U^{2}}{2} \chi_{\mathbf{q}}^{o}\left(i \nu_{m}\right)\left(\frac{3}{1-U \chi_{\mathbf{q}}^{o}\left(i \nu_{m}\right)}+\frac{1}{1+U \chi_{\mathbf{q}}^{o}\left(i \nu_{m}\right)}-2\right) \text {. }
$$

Here, $\chi_{\mathbf{q}}^{o}\left(i \nu_{m}\right)=-\frac{T}{N} \sum_{\mathbf{k}, n} G_{\mathbf{k}+\mathbf{q}}\left(i \omega_{n}+i \nu_{m}\right) G_{\mathbf{k}}\left(i \omega_{n}\right)$ is the particle-hole bubble and $\omega_{n}=$ $(2 n+1) \pi T$ and $\nu_{m}=2 m \pi T$ are the fermionic and bosonic Matsubara frequencies, respectively. Furthermore, the Greens function is given by the Dyson equation $G_{\mathbf{k}}\left(i \omega_{n}\right)=$ $\left(i \omega_{n}+\mu-\varepsilon(\mathbf{k})-\Sigma_{\mathbf{k}}\left(i \omega_{n}\right)\right)^{-1}$ where $\varepsilon(\mathbf{k})=-2 t\left(\cos \left(k_{x}\right)+\cos \left(k_{y}\right)\right)$ is the free dispersion. These equations are analytically transformed to the real frequency axis yielding a set of equations for the Greens function $G_{\mathbf{k}}(\omega)$ and the self-energy $\Sigma_{\mathbf{k}}(\omega)$, which is solved self consistently 22 .

In Fig. 1(a) we show our results for the spectral density $\varrho_{\mathbf{k}}(\omega)=-\frac{1}{\pi} \operatorname{Im} G_{\mathbf{k}}(\omega)$ for various $\mathbf{k}$-values shifted by $\mathbf{Q}$ with respect to the main FS as can be seen in the inset, where we 
plot the first quadrant of the Brillouine zone. The doping concentration $x=1-n$, with occupation number per site $n$, is given by $x=0.12$. Besides the main peak with a position similar to that of an uncorrelated system, we find new states below the Fermi surface. These are the shadow states which occur for $\mathbf{k}$-values where one expects the states of the new band of a long range ordered antiferromagnet, although our calculation was performed in the paramagnetic state. Furthermore, an estimate of the antiferromagnetic correlation length from the spin spin susceptibility, neglecting vertex corrections, yields $\xi \approx 2.5$ lattice spacings, in agreement with neutron scattering experiments 13 . These results show that indeed spin fluctuation induced shadow states without long range antiferromagnetic order and with short correlation length exist. 0 The distance of these states with respect to the Fermi energy is always finite, i.e. they never cross the Fermi energy, but are of the order of the experimental resolution of a photoemission experiment.

In Fig. 1(b) we present our results for the doping dependence of $\varrho_{\mathbf{k}}(\omega)$ at the FS-shadow near the $\mathbf{k}$-value $(\pi, 0)$. One clearly recognizes the strong increase of the shadow band intensity for decreasing doping concentration. Consequently, an anomalous occupation of states with momentum outside the FS occurs. Besides the intensity variation, important information about the dynamical character of the antiferromagnetic correlations can be obtained from the position of these new states. Therefore, we consider the quasi particle dispersion obtained from the momentum variation of all local maxima of $\varrho_{\mathbf{k}}(\omega)$.

In Fig. 2 we show our results for the quasi particle dispersion (solid line and squares) for different doping values in comparison with the dispersion of the corresponding uncorrelated systems (dotted line) for $\mathbf{k}$-values indicated in the inset. The solid line refers to the global maximum and shows the main band dispersion, whereas the shadow states are indicated by the squares. Most interestingly, one observs for decreasing doping a reduction of the lowest distance $\omega_{\text {exc }}$ of a shadow state with respect to the Fermi energy. For $\omega_{\text {exc }} \rightarrow 0$, we expect a static antiferromagnetic order. Therefore, we interpret the inverse of $\omega_{\text {exc }}$ as a measure of the life time of the dynamically symmetry broken state. Another precursor effect of the antiferromagnetic state in the paramagnetic phase is the opening of a spin density gap, as 
can be seen from the strong deformation of the main band (solid line) upon doping. For larger doping, solely a flattening of the dispersion near the Fermi level occurs, whereas a sudden jump near the magnetic Brillouine zone border $(\pi, 0)$ can be seen for $x=0.12$ and $x=0.09$. Consequently, on a finite time scale, a quasi particle cannot distinguish the actual state from that of a long range ordered phase. Therefore, the system behaves on shorter time scales as in a symmetry broken state, although no antiferromagnetic order occurs in the thermodynamic limit, where each excitation has infinite time to relax into equilibrium. This point of view is supported by the experimental observation by Osterwalder et al.14 where the shadow states seem to be more sharp for an energy $\omega=60 \mathrm{meV}$ than directly at the Fermi level.

In Fig. 3, we present our results for the doping dependence of the "van Hove" scale $\omega_{\mathrm{vH}}$, i.e. the main band energy for $\mathbf{k}=(\pi, 0), \omega_{\mathrm{exc}}$ and the relative intensity of the shadow states with respect to the main band height. Comparing $\omega_{\mathrm{vH}}$ with the corresponding energy for $U=0$, we find that due to the electronic correlations states are shifted towards the Fermi level, and our results for $x \approx 0.16$ become comparable with the experimental value of $30-50 \mathrm{meV}$. For lower doping values, a saturation of $\omega_{\mathrm{vH}}$, related to the opening of a spin density gap, can be observed. Furthermore, the close relation between the variations of the intensity of the shadow states and of $\omega_{\text {exc }}$ can be seen in Fig. 3 (b) and (c). Between $x=0.14$ and $x=0.12$ the abrupt decrease of $\left|\omega_{\text {exc }}\right|$ occurs simultaneously with the strong increase of the shadow band intensity. Consequently, we find that the shadow band phenomenon appears suddenly below a critical doping concentration $(x \approx 0.14)$. Note that this might also be related to the optimal doping $x_{\mathrm{opt}}=0.13$ of the superconducting transition temperature $T_{c}$, which was found by us in a corresponding FLEX-calculation in the superconducting state 15.

Although all our calculations are performed using a model dispersion, we believe that the occurrence of shadow states is of more general character. The two important prerequisites for these states are a flat dispersion of the quasiparticles near the Fermi energy and flat portions of the Fermi surface, i.e. FS-nesting. These features are experimentally observed 
in various cuprate systems 16 and are also a basic content of more realistic parametrizations of band structure calculations 17 . While FS-nesting favors the antiferromagnetic correlations, flat bands are necessary because the states with momentum $\mathbf{k}$ and $\mathbf{k}+\xi^{-1}$, which contribute both to a shadow state at $\mathbf{k}+\mathbf{Q}$, have to be energetically close enough together to ensure a well defined peak in $\varrho_{\mathbf{k}+\mathbf{Q}}(\omega)$. Even if a change of the model dispersion and the FS leads to less pronounced nesting, a correlation induced flattening of the bands can compensate this with respect to the shadow state intensity. It is important to remark that our theory yields for larger values of the ratio $U / t$ an increased range of flat bands in $\mathbf{k}$-space. For $U / t=6$ and $x=0.12$ the quasi particle energy for $\mathbf{k}=(5 \pi / 8,0)$ is $80 \mathrm{meV}$ in comparison with $130 \mathrm{meV}$ for $U / t=4$. Consequently, we believe that the moderate values of $U / t$ of our calculation give a lower bound for the shadow band intensity for the model dispersion under consideration.

In conclusion, the doping dependence of spin fluctuation induced shadow states was investigated using a self consistent description for the dynamical excitation spectrum of the 2D one band Hubbard model within the FLEX-approximation. It was shown that below a critical doping concentration $x \approx 0.14$ these states appear suddenly simultaneously with a sharp decrease of their lowest energy distance $\omega_{\text {exc }}$ with respect to the Fermi energy. The additional opening of a spin density gap makes it impossible to discriminate on finite time scales (shorter than $\omega_{\text {exc }}$ ) this paramagnetic state with strong dynamical spin fluctuations from a long range ordered state. All this demonstrates the strong influence of short range antiferromagnetic correlations on the excitation spectrum of these compounds and might be stimulating for further experimental investigations of the shadow band phenomenon. 


\section{REFERENCES}

${ }^{1}$ P. Aebi, J. Osterwalder, P. Schwaller, L. Schlapbach, M. Shimoda, T. Mochiku, and K. Kadowaki, Phys. Rev. Lett. 72, 2757 (1994).

2 S. Chakravarty, Phys. Rev. Lett. 74, 1885 (1995).

${ }^{3}$ P. Aebi et al., Phys. Rev. Lett. 74, 1886 (1995).

${ }^{4}$ A. P. Kampf and J. R. Schrieffer, Phys. Rev. B 42, 7967 (1990).

${ }^{5}$ M. Langer, J. Schmalian, S. Grabowski, and K.-H. Bennemann, Phys. Rev. Lett 75, 4508 (1995).

${ }^{6}$ S. Haas, A. Moreo, and E. Dagotto, Phys. Rev. Lett 74, 4281 (1995).

7 T. Imai, C. P. Slichter, K. Yoshimura, and K. Kosuge, Phys. Rev. Lett. 70, 1002 (1993).

${ }^{8}$ R. J. Birgenau et.al., Phys. Rev. B 38, 6614 (1988).

${ }^{9}$ N. E. Bickers, D. J. Scalapino, Ann. Phys. (N.Y.) 193, 206 (1989).

${ }^{10}$ N. E. Bickers, D. J. Scalapino, and S. R. White, Phys. Rev. Lett. 62, 961 (1989).

${ }^{11}$ G. Baym and L. P, Kadanoff, Phys. Rev. 124, 287 (1961); G. Baym ibid. 127, 1391 (1962).

12 The results presented here were calculated on a lattice of size $64 \times 64$ lattice sites and with an energy resolution of 4096 points in the interval $[-30 t, 30 t]$, for details see J. Schmalian, M. Langer, S. Grabowski, and K.-H. Bennemann, to appear in Computer Physics Communications.

13 J. M. Tranquada et al., Phys. Rev. B 46, 5561 (1992).

${ }^{14}$ J. Osterwalder, P. Aebi, P. Schwaller, and L Schlappbach, Applied Physics A 60, 247 (1995).

${ }^{15}$ S. Grabowski, J. Schmalian, M. Langer, and K.-H. Bennemann (preprint). 
16 Z. X. Shen and D. S. Dessau, Phys. Rep. 253, 1 (1995).

${ }^{17}$ O. K. Andersen, A. I. Liechtenstein, O. Jepsen, and F. Paulsen, to be published in Journal of Physics and Chemistry of Solids. 


\section{FIGURES}

FIG. 1. Spectral density of the shadow states for (a) doping concentration $x=0.12$ and various $\mathbf{k}$-values shifted by $\mathbf{Q}$ with respect to the main Fermi surface and (b) for $\mathbf{k}$ on the FS-shadow closest to $(\pi, 0)$ and for different doping values. The inset indicates the main Fermi surface (solid line) its shadow (dashed line) and the $\mathbf{k}$-values of part (a) in the first quadrant of the Brillouine zone.

FIG. 2. Quasi partice dispersion of the main band (solid line) and the shadow states (squares) for different doping values and for $\mathbf{k}$-values indicated in the inset. The dashed line refers to the dispersion for $U=0$. Note the pronounced deformation of the main band dispersion and the decrease of the lowest energy distance of a shadow state with respect to the Fermi energy $(\omega=0)$ for decreasing doping.

FIG. 3. Doping dependence (a) of the main band energy $\omega_{\mathrm{vH}}$ for $\mathbf{k}=(\pi, 0)$ in comparison with its value for $U=0$, (b) of the lowest energy distance $\omega_{\text {exc }}$ of a shadow state with respect to the Fermi energy, and (c) of the relative intensity of the shadow state with respect to the main band height closest to $(\pi, 0)$. Note the simultaneous and sudden appearance of large shadow band intensities and low $\omega_{\text {exc }}$ for doping values below $x=0.14$. 

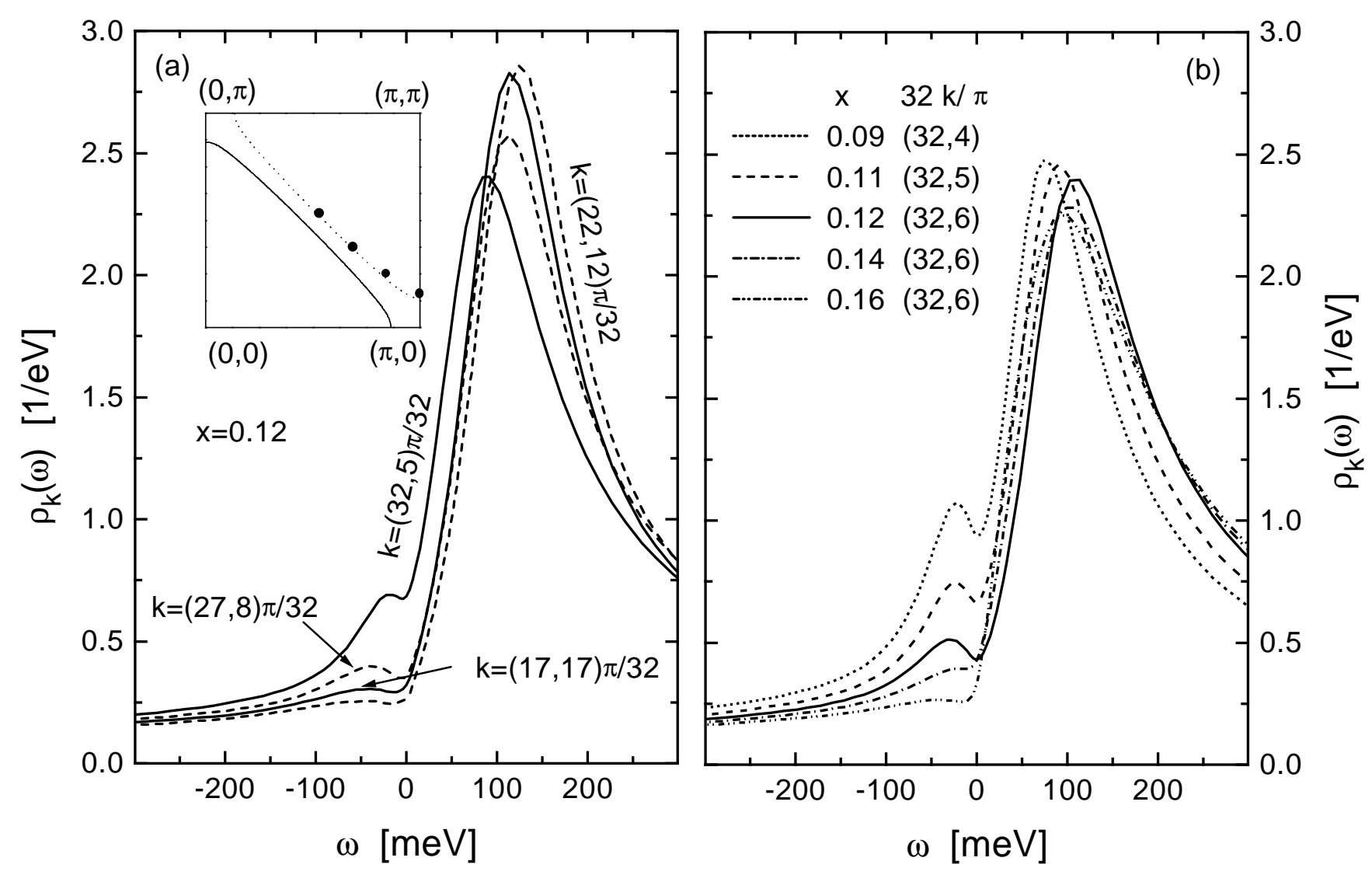

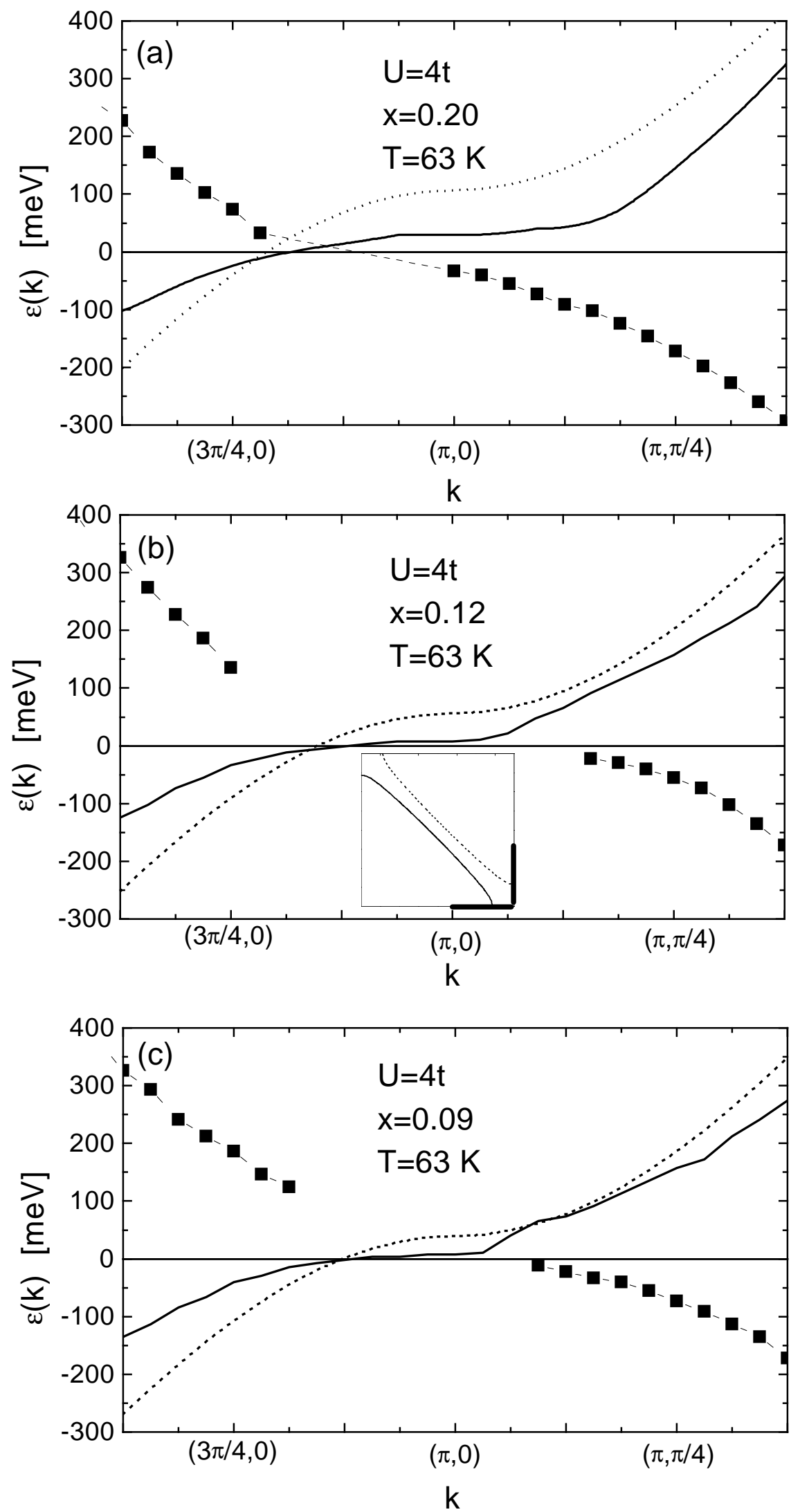

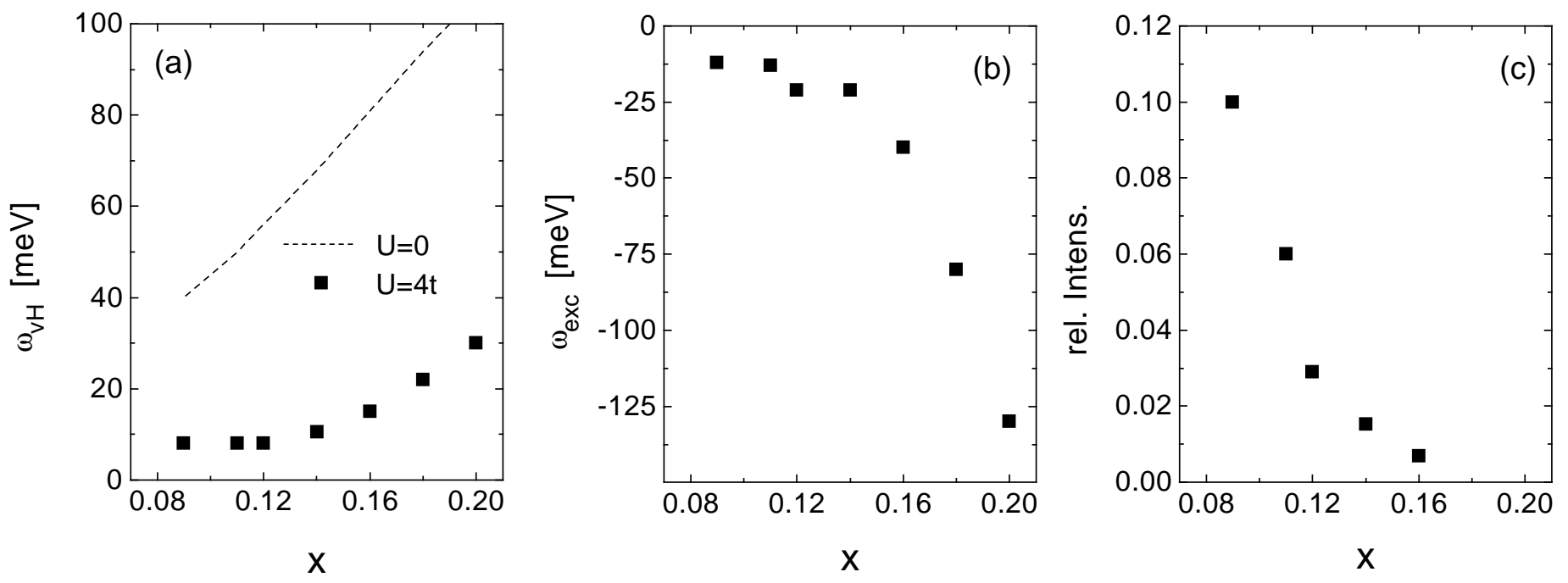